\title{
Clinical correlates of angiographically diagnosed idiopathic pulmonary hypertension
}

\author{
Huon H Gray, John M Morgan, Ian H Kerr, Graham A H Miller
}

\begin{abstract}
During 1970-87 43 patients with unexplained pulmonary hypertension (mean pulmonary arterial pressure $>25 \mathrm{~mm}$ Hg) were admitted to the Brompton Hospital and classified by angiographic criteria as having either symmetrical peripheral pulmonary artery pruning (thought to represent primary plexogenic pulmonary arteriopathy), $n=21$, or asymmetrical pulmonary arterial occlusions (thought to represent chronic thromboembolic disease), $\mathbf{n}=22$. Patients with symmetrical pulmonary arteriopathy had significantly higher mean pulmonary arterial pressures $(67 \mathrm{~mm} \mathrm{Hg})$ at the time of presentation than those with asymmetrical pulmonary arteriopathy $(49 \mathrm{~mm} \mathrm{Hg})$. Clinical distinction between these two groups was impossible. Survival from the time of diagnosis was similarly poor in the two groups (26 weeks and 38 weeks) and did not correlate with any of the haemodynamic measurements. The difficulties in making distinctions between these conditions are discussed.
\end{abstract}

Chronic pulmonary hypertension may be due to various cardiac or pulmonary conditions, ${ }^{1}$ the causes of which are often apparent clinically or as a result of investigations. "Primary" pulmonary hypertension is a term that has often been used synonymously with "clinically unexplained pulmonary hypertension," and this confusing terminology ${ }^{2}$ has to some extent persisted despite attempts by the World Health Organisation to be more specific by subdividing unexplained pulmonary hypertension into three pathological categories $^{3}$ : (1) primary plexogenic pulmonary arteriopathy; (2) recurrent thromboembolic pulmonary hypertension; and (3) pulmonary veno-occlusive disease. The last, being extremely rare, is excluded from our series and will not be discussed further.

Clinically it is impossible to distinguish these conditions, ${ }^{4}$ though a clear history of prior repeated episodes of deep venous thrombosis or pulmonary embolism suggests a thromboembolic cause in a few cases..$^{5}$ As the World Health Organisation's classification ${ }^{3}$ is based on pathological findings lung biopsy would seem the obvious means of attempting to make a diagnosis during life. Transbronchial biopsy specimens, however, often provide insufficient tissue for analysis ${ }^{6}$ and, although some have recommended open lung biopsy, ${ }^{7}$ many regard this procedure and the attendant anaesthesia as hazardous. ${ }^{68}$ Even when tissue is available for analysis pathological differentiation is not always easy.

As an alternative to lung biopsy we have used angiographic criteria in an attempt to distinguish these conditions during life. On this basis we identified 21 patients with symmetrical pulmonary arteriopathy (thought to represent primary plexogenic pulmonary arteriopathy) and 22 with asymmetrical pulmonary artery occlusions (thought to represent chronic thromboembolic pulmonary hypertension) who were investigated for idiopathic pulmonary hypertension during 1970-87. We have analysed the clinical and investigative findings in an attempt to determine whether such an angiographic differentiation allows clinical differences to be identified.

\section{Patients and methods}

During the years 1970-87 43 patients admitted to the Brompton Hospital had pulmonary hypertension confirmed and had no systemic, hepatic, pulmonary, or congenital cardiac disorder to explain the finding. ${ }^{9}$ A diagnosis of pulmonary hypertension was suspected on clinical evidence and confirmed by the finding of a mean pulmonary artery pressure above $25 \mathrm{~mm} \mathrm{Hg}$. Patients with dilated proximal pulmonary arteries and symmetrical peripheral pulmonary arterial "pruning" without filling defects were considered to have primary plexogenic pulmonary arteriopathy, whereas asymmetrical scattered arterial occlusions or proximal major arterial filling defects were regarded as suggesting chronic thromboembolism. ${ }^{10}$ We accept that this angiographic distinction may be arbitrary and may not classify patients into the same categories as other methods, such as histological analysis of lung tissue. ${ }^{11}$

Our intention was to determine whether angiographic differentiation defined patients with different clinical courses. So that we can be more precise, and not assume that the arteriographic findings reflect histological differences, we refer to the two groups of patients in this paper according to their radiological abnormalities (symmetrical and asymmetrical pulmonary arteriopathy) rather than the World Health Organisation groupings, which they are thought to represent (primary plexogenic and chronic thromboembolic pulmonary hypertension). The
Address for reprint requests: Dr HH Gray, Wessex Unit, Southampton General Hospital, Southampton SO9 4XY.

Accepted 14 March 1990 
clinical features, investigative findings, and subsequent survival of these patients were assessed and the exact probability test, Mann-Whitney test, and Spearman's rank correlation were used for statistical analysis.

\section{Results}

\section{THE PATIENTS}

The mean age at presentation of the 21 patients with symmetrical pulmonary arteriopathy (five male, 16 female) was 39 (range 9-62) years and that of the 22 patients with asymmetrical pulmonary arteriopathy (nine male, 13 female) was 46 (range 15-68) years. Although patients with symmetrical pulmonary arteriopathy were younger and more likely to be female these differences were not significant. One woman with asymmetrical and two with symmetrical pulmonary arteriopathy had taken the contraceptive pill within two years of diagnosis and two in each group had been pregnant during this period. No patient was prescribed the contraceptive pill or became pregnant after diagnosis.

Of the patients with symmetrical pulmonary arteriopathy, two were current smokers, one was taking fenfluramine at the time of diagnosis, one had Raynaud's disease, and two had mothers who died of unknown causes when relatively young ( 38 and 40 years). Of those diagnosed as having asymmetrical pulmonary arteriopathy, three were current smokers, one had a clinical diagnosis of mild rheumatoid arthritis, and one had a mother who had died after a pulmonary embolism. No other relevant factors emerged from direct questioning. Presentation in childhood was unusual in both groups.

\section{SYMPTOMS (table 1)}

Symptoms were similar in the two groups. All patients had exertional dyspnoea and reduced exercise tolerance. Pleuritic chest pain and haemoptyses occurred at some stage during the presenting illness more frequently in those with asymmetrical (five, 23\%) than sym-

Table 1 Clinical data in 21 patients with symmetrical (SPA) and 22 patients with asymmetrical ( $A P A)$ pulmonary arteriopathy (all differences non-significant)

\begin{tabular}{lcc}
\hline & $S P A$ & $A P A$ \\
& No $(\%)$ & No $(\%)$ \\
\hline CLINICAL HISTORY & & \\
$\begin{array}{l}\text { Present } \\
\text { Dyspnoea }\end{array}$ & $21(100)$ & $22(100)$ \\
$\quad$ Exertional chest pain & $2(9 \cdot 5)$ & $4(18)$ \\
Past & & \\
$\quad$ Pleuritic pain & $1(5)$ & $5(23)$ \\
Haemoptysis & $1(5)$ & $5(23)$ \\
Deep vein thrombosis & $0(0)$ & $2(9)$ \\
Pulmonary embolism & $0(0)$ & $1(4 \cdot 5)$ \\
Syncope & $4(19)$ & $1(4.5)$ \\
& & \\
CLINICAL signs & $19(90)$ & $19(86)$ \\
Loud pulmonary 2nd sound & $6(29)$ & $7(32)$ \\
Right ventricular S3 & $3(14)$ & $2(9)$ \\
Tricuspid regurgitation & $1(5)$ & $1(4.5)$ \\
Pulmonary regurgitation & $10(48)$ & $8(36)$ \\
Raised jugular venous pressure & $3(14)$ & $2(9)$ \\
Peripheral oedema & $4(19)$ & $2(9)$ \\
Liver enlargement & & \\
\hline
\end{tabular}

metrical arteriopathy (one, $5 \%$ ), as did a past history (at any time) of deep venous thrombosis (two, 9\%, and none) or pulmonary embolism (one, 4.5\%, and none). These differences did not, however, reach significance. The patient who had had a clinically recognised pulmonary embolus did not have associated haemodynamic impairment at the time, suggesting that it had been an acute minor rather than massive pulmonary embolus. In those with symmetrical pulmonary arteriopathy syncope was the most common single symptom (four, $19 \%$ ) after exertional fatigue and dyspnoea, occurring more frequently (though the difference was nonsignificant) in this group than in those with asymmetrical pulmonary arteriopathy (one, $4.5 \%$ ).

\section{DURATION OF SYMPTOMS}

Although the onset of symptoms does not necessarily reflect the onset of disease, the duration of symptoms at the time of presentation was used as a rough guide to the chronicity and rapidity of progression of the condition. The mean duration of symptoms for the two groups was the same (symmetrical pulmonary arteriopathy 23 weeks, range 3-84, and asymmetrical disease 23 weeks, range 284).

\section{CLINICAL SIGNS}

An accentuated pulmonary component of the second heart sound, compatible with pulmonary hypertension, was found with equal frequency in patients with symmetrical (19, $90 \%$ ) and asymmetrical $(19,86 \%)$ pulmonary arteriopathy. Other physical findings were less frequent but were seen equally in the two groups (table 1). No symptom or sign or combination of these was capable of distinguishing between the two conditions.

\section{ELECTROCARDIOGRAPHY}

All patients were in sinus rhythm. Evidence of right ventricular hypertrophy or strain pattern or both was seen in $20(95 \%)$ of the patients with symmetrical and $19(86 \%)$ of those with asymmetrical pulmonary arteriopathy. One patient with asymmetrical disease had right bundle branch block. There were no other abnormalities.

\section{RADIOGRAPHY}

All patients had plain posteroanterior chest radiography and the films were reviewed by an experienced radiologist. Enlargement of the main pulmonary arteries was equally frequent in patients with symmetrical (19, $90 \%)$ and asymmetrical $(19,86 \%)$ pulmonary arteriopathy. An increased cardiothoracic ratio was present in seven $(33 \%)$ and six $(27 \%)$ and peripheral oligaemia in four $(19 \%)$ and four $(18 \%)$ respectively. The chest radiograph proved helpful in making a diagnosis of pulmonary hypertension but not in distinguishing between the two groups. Neither venography nor radionuclide perfusion lung scanning was performed sufficiently frequently for us to make any comment on the value of the techniques in the investigation of pulmonary vascular disease. 
Table 2 Pressures, cardiac output, and total pulmonary vascular resistance (TPR) at right heart catheterisation in 21 patients with symmetrical and 22 patients with asymmetrical pulmonary arteriopathy (means with ranges and standard deviations in parentheses)

\begin{tabular}{|c|c|c|c|}
\hline & Symmetrical & & Asymmetrical \\
\hline \multicolumn{4}{|l|}{ Pressure $(\mathrm{mm} \mathrm{Hg})$} \\
\hline Right atrial & $11(6-27,6 \cdot 4)$ & NS & $11(5-20,4 \cdot 6)$ \\
\hline Right ventricular systolic & $95(75-134,18 \cdot 9)$ & $\star \star \star$ & $66(50-98,20.0)$ \\
\hline Mean pulmonary arterial & $67(43-79,17 \cdot 0)$ & $\star \star$ & $49(40-60,16 \cdot 2)$ \\
\hline Cardiac output $\left(1 \mathrm{~min}^{-1}\right)$ & $2 \cdot 49(1 \cdot 9-3 \cdot 1.0 \cdot 5)$ & NS & $2 \cdot 18(1 \cdot 8-4 \cdot 0,0 \cdot 35$ \\
\hline Mean TPR (TPR units) & $27(13 \cdot 7-49 \cdot 5,10 \cdot 5)$ & NS & $23(10-39 \cdot 4,8 \cdot 2)$ \\
\hline
\end{tabular}

RIGHT HEART CATHETERISATION (table 2)

The two groups had similar right atrial pressures but those with symmetrical pulmonary arteriopathy had significantly higher mean pulmonary arterial pressures $(p<0.01)$. Total pulmonary resistance was calculated as the mean pulmonary arterial pressure divided by cardiac output, cardiac output being measured by the indirect Fick method with an assumed basal oxygen consumption. Total pulmonary resistance was similar in patients with symmetrical (27 Wood units) and asymmetrical (23 Wood units) pulmonary arteriopathy as cardiac output was higher in those with symmetrical disease. Pulmonary arteriography was performed in all patients and 19 arteriograms were still available for analysis. These were reviewed, without knowledge of any clinical details, by the same radiologist (IHK) who had reported on them previously; his reports agreed in all cases with the original ones. Thus the radiological features used to differentiate these conditions were identified consistently and the original report is unlikely to have been influenced by clinical details on the radiography request form.

\section{FOLLOW UP DATA}

Survival was calculated from the date of presentation to the Brompton Hospital to the date of death or, if the patient was still alive, to the end of 1987. By adding this to the duration of symptoms before presentation for the patients who died an estimate could be made of total survival from the onset of symptoms, the assumption being that the onset of symptoms was related to the onset of the disease. In this way we hoped to obtain information on the progress of these conditions and their likely prognosis.

DEATHS

At the end of 1987 three of the original 43 patients (all with asymmetrical pulmonary arteriopathy) had been lost to follow up. Of those for whom data were available, 19 of 21 patients with symmetrical (four male, 15 female) and 16 of 20 patients with asymmetrical disease (eight male, eight female) were known to have died. All patients were thought to have died as a direct consequence of their pulmonary hypertension; the usual mode of death was right heart failure. The mean survival time from the onset of symptoms to death was 47 (range 7-120) weeks for those with symmetrical and 72 (range 7-204) weeks for those with asymmetrical pulmonary arteriopathy.
Survival from the time of diagnosis to death was 26 (range 1-88) and 38 (range 1-192) weeks. Patients with asymmetrical disease therefore tended to live longer but this difference failed to reach significance $(\mathrm{p}=0 \cdot 12)$. There was a significant positive correlation for both groups between the duration of symptoms before presentation and the time from presentation to death $(r=0.7$ for symmetrical and $r=0.8$ for asymmetrical disease), such that patients with a longer history of symptoms before presentation tended to live longer after diagnosis. This suggests that the duration of previous symptoms will provide a guide to prognosis when a patient first presents. There was no correlation between survival time from the onset of symptoms or from the date of presentation and any of the measured haemodynamic variables. Thus prognosis could not be predicted reliably from the severity of pulmonary hypertension. There were no significant differences in survival between the sexes in either group.

Postmortem examinations were performed in only $34 \%$ of those who died (four with symmetrical and eight with asymmetrical pulmonary arteriopathy). The rarity of these conditions meant that the patients were referred from various parts of the United Kingdom and death often occurred in a local hospital or at home. Necropsies were often refused by relatives or not requested by medical staff and when they were performed seemed to do little more than confirm the presence of severe pulmonary vascular disease. It proved impossible to obtain any further information or samples of the material submitted for histological examination for review.

\section{SURVIVORS}

Of the 40 patients for whom follow up data were available, two from the symmetrical and three from the asymmetrical group were still alive at the end of 1987 . They had survival times from the onset of symptoms of 7-24 and 26-40 weeks, and from the time of diagnosis of 4-21 and 9-26 weeks.

\section{EFFECTS OF TREATMENT}

All patients with asymmetrical and three with symmetrical pulmonary arteriopathy had oral anticoagulant treatment (warfarin) and seven of those with symmetrical disease had vasodilator treatment. The uncontrolled nature of the decision to treat makes it impossible to determine whether treatment had any influence on survival.

\section{Discussion}

Of all the methods used to categorise patients with unexplained pulmonary hypertension, histological analysis of lung tissue has received the most widespread acceptance, as shown by its adoption by the World Health Organisation. $^{3}$ Histological classification may, however, be an imperfect "gold standard." Plexiform lesions are seen in patients with other forms of pulmonary hypertension ${ }^{21213}$ but in the absence of intracardiac or extra- 
cardiac shunts are described as a mainstay in the diagnosis of primary plexogenic pulmonary arteriopathy. ${ }^{11}$ But plexiform lesions may be confused with organised thrombi ${ }^{14}$ and in primary plexogenic pulmonary arteriopathy thrombus may occur in situ, giving an appearance similar to that seen with chronic thromboembolic disease. ${ }^{11}$ Histological differentiation is easier and more reliable in the earlier stages of pulmonary vascular disease.

Examples of the difficulty in classifying patients when alive correctly are provided by two studies. In one of these a large group of patients diagnosed clinically and angiographically as having primary plexogenic pulmonary hypertension were reviewed and those with any evidence of pulmonary embolism or deep venous thrombosis were excluded. Despite this exclusion, in over half of the necropsies performed plexogenic lesions were absent and thrombus was the major histological abnormality. ${ }^{15}$ Similarily, in the largest pathological series $20^{\circ}$ of patients with an antemortem clinical diagnosis of primary plexogenic pulmonary arteriopathy had histological evidence of thromboembolic disease at necropsy. ${ }^{11}$ Further difficulties are encountered when attempts are made to obtain samples of lung tissue during life ${ }^{7}$ because samples obtained transbronchially are often too small to be definitive ${ }^{6}$ and larger amounts of tissue may only be obtainable by open lung biopsy, which may be hazardous. ${ }^{8}$ Moreover, single biopsy samples may not be representative of the lung as a whole. ${ }^{4}$ We do not intend to imply that such problems with histological analysis invalidate its use in distinguishing these conditions. Histology is probably still the most accurate means of assessing the nature of pulmonary vascular disease; but the area of overlap, where histological appearances may be difficult to interpret, raises the possibility that there may be a spectrum of disease rather than two well defined conditions.

The argument for using angiography to classify patients with unexplained pulmonary hypertension is that it is probably less hazardous than lung biopsy and is able to show vessels of sufficient size from the whole of both lungs, especially when magnified views are used, ${ }^{4}$ rather than the inevitably small areas available from biopsy samples. The same argument favours radionuclide perfusion lung scanning: scans have been shown to be normal in primary plexogenic pulmonary arteriopathy ${ }^{16}$ but may be abnormal in chronic thromboembolic disease, ${ }^{413}$ especially in patients with unresolved emboli affecting large rather than small pulmonary arteries. ${ }^{17}$ These patients must be identified because some may be suitable for pulmonary thrombectomy and endarterectomy, ${ }^{18}$ especially those who have adequate distal pulmonary perfusion via collateral vessels shown by bronchial arteriography. ${ }^{19}$

There is clearly little point in classifying patients angiographically unless such a classification either correlates well with lung biopsy appearances (which we have not had the data to assess) or defines groups of patients with different clinical outcomes. Whether angiographic classification of patients is the same as that based on histological analysis of necropsy or biopsy material is still not clear-hence our use of radiological abnormalities (symmetrical and asymmetrical pulmonary arteriopathy) to refer to the two groups of patients rather than the histological classifications (primary plexogenic and chronic thromboembolic pulmonary hypertension) that they may represent. There are three main reasons why the correlation between angiographic and histological features of idiopathic pulmonary hypertension remains unresolved. Firstly, there has been a reluctance in the past to perform pulmonary arteriography in patients with pulmonary hypertension because of reports of sudden death, ${ }^{20}$ though most of these cases occurred many years ago and more recent experience with primary plexogenic pulmonary arteriography, ${ }^{41}$ chronic thromboembolic pulmonary hypertension, ${ }^{4}$ and massive pulmonary embolism ${ }^{22}$ has shown the procedure to be safe. Secondly, in the larger series where angiography has been used to help to classify patients either the arteriograms from the two groups have not always been compared $^{15}$ or the angiographic criteria used to make such a classification have not always been defined. ${ }^{91617}$ Thirdly, no study has been performed that directly compares angiographic and histological findings in the same patients with unexplained pulmonary hypertension. All these factors make retrospective analysis of reported series difficult. In two clinical studies the arteriograms of patients with unexplained pulmonary hypertension were compared and shown to separate patients into different groups, the groupings correlating fairly well with diagnoses made by clinical criteria ${ }^{23}$ or perfusion lung scanning. ${ }^{13}$ In our series the available pulmonary arteriograms were reviewed blindly by one of us (IHK) and the results found to be $100 \%$ concordant with the previous reports, showing that the angiographic criteria were at least consistent. Whether these criteria, any more than histological ones, truly identify two distinct diseases remains uncertain.

We and others ${ }^{415}$ have been unable to identify any clinical features that distinguish between these two angiographic groups of patients, though some trends were noted in our results. Patients with symmetrical pulmonary arteriography were more likely to be female and those with asymmetrical disease were older and had less severe pulmonary hypertension at the time of presentation and appeared to live longer. The duration of symptoms before diagnosis in both groups was positively correlated with length of survival. Although some have shown a relation between survival and haemodynamic measurements at presentation, ${ }^{24}$ none of our haemodynamic data showed any correlation with survival. To our knowledge only one group of workers has classified patients with idiopathic pulmonary hypertension on angiographic grounds and compared the clinical features of the two groups, and their results were similar to ours. ${ }^{4}$ Our inability to find clinical differences may 
have been due to the relatively small number of patients available for analysis, or the clinical consequences in the two groups might have been truly indistinguishable even if larger numbers had been available. It is also possible that the two angiographic groups do not represent genuinely distinct pathophysiological entities. $^{813}$

Although there is clearly some difficulty in subdividing patients with idiopathic pulmonary hypertension, by whatever means, one could argue that this is of little importance as the prognoses of these subgroups (such as primary plexogenic and chronic thromboembolic pulmonary hypertension) are regarded as similarly poor. ${ }^{15}$ Apart from a natural desire for diagnostic accuracy, however, there are other reasons for wishing to distinguish between them. If more reliable differentiation could be achieved more effective treatment might be identified and prognoses altered, though at present there are no prospective data to suggest that any drug treatment alters the course of either disease. One retrospective analysis has suggested an improved survival when patients with either primary plexogenic or chronic thromboembolic pulmonary hypertension were treated with anticoagulants. ${ }^{15}$ Patients with primary plexogenic pulmonary arteriopathy may be symptomatically improved by vasodilators but there is still no evidence that vasodilators improve prognosis (see ref 8 for review), though this may change when data from trials of longer term prostacyclin infusions are available. ${ }^{2526} \mathrm{~A}$ favourable haemodynamic response to short term vasodilator treatment, however, may identify those patients with a more reactive pulmonary vasculature and a better prognosis. ${ }^{27}$ Diagnostic accuracy may thus allow classification at an earlier stage in the disease, when-at least in the case of primary plexogenic pulmonary arteriopathy-it may respond better to treatment. All patients with end stage unexplained pulmonary hypertension, irrespective of aetiology, should be considered for heart-lung transplantation; but the potential benefit of thrombectomy and endarterectomy for suitable patients with chronic thromboembolic diseas $^{1819}$ is a stimulus to investigation of these patients. The indication for inferior vena caval plication or insertion of filter devices in patients thought to have chronic thromboembolic pulmonary hypertension is disputed. ${ }^{28-31}$ In view of the high morbidity and mortality of these procedures, ${ }^{31}$ and of embolic recurrences despite their use, our view has been that they are inappropriate. We have used long term anticoagulant treatment in the hope of reducing the likelihood of further emboli.

If our understanding of idiopathic pulmonary hypertension is to be increased it seems sensible for patients to be included in a national registry ${ }^{9}$ and for as many as possible to be referred to a few centres so that experience can be concentrated and prospective research into their aetiology, diagnosis, and treatment advanced. A prospective multicentre controlled trial of the investigation and treatment of these rare conditions may help considerably to improve their management.

1 Michael JR, Summer WR. Pulmonary hypertension. Lung 1985;163:65-82.

2 Haworth SG. Primary pulmonary hypertension. Br Heart $J$ 1983;49:517-21.

3 Hitano S, Strasser T, eds. Primary pulmonary hypertension: report on a WHO meeting. Geneva: World Health Organisation, 1975 .

4 D'Alonzo GE, Bower JS, Dantzker DR. Differentiation of patients with primary and thromboembolic pulmonary hypertension. Chest 1984;85:457-61.

5 Riedel M, Prerovsky I, Stanek V, Ressl J, Stadlerova V, Widimsky J. Chronic thromboembolic disease. Long term follow up. Prog Respir Res 1980;13:134-40.

6 Oakley C. Management of primary pulmonary hypertension. Br Heart J 1985;53:1-4.

7 Wagenvoort CA. Lung biopsy specimens in the evaluation of pulmonary vascular disease. Chest 1980;77:614-25.

8 Hughes JD, Rubin LJ. Primary pulmonary hypertension. An analysis of 28 cases and a review of the literature. Medicine (Baltimore) 1986;65:56-72

9 Rich S, Dantzker R, Ayres SM, et al. Primary pulmonary hypertension. A national prospective study. Ann Intern Med 1987;107:216-23.

10 Chrispin AR, Goodwin JF, Steiner RE. The radiology of obliterative pulmonary hypertension and thromboembolobliterative pulmonary hypertens
ism. $B r J$ Radiol 1963;36:705-14.

11 Wagenvoort CA, Wagenvoort N. Primary pulmonary hypertension. A pathologic study of the lung vessels in 156 clinically diagnosed cases. Circulation 1970;42:1163-84.

12 Editorial. A better outlook in primary pulmonary hyperten sion. Lancet 1986; i:1420-1.

13 Wilson AG, Harris CN, Lavender JP, Oakley CM. Perfusion lung scanning in obliterative pulmonary hypertension. Br Heart J 1973;35:917-30.

14 Bjornsson J, Edwards WD. Primary pulmonary hypertension: A histopathologic study of 80 cases. Mayo Clin Proc sion: A histopath

15 Fuster V, Steele PM, Edwards WD, Gersh BJ, McGopon $M D$, Frye RL. Primary pulmonary hypertension: natural history and the importance of thrombosis. Circulation 1984;70:580-7.

16 Rozkovec A, Montanes P, Oakley CM. Factors that influence the dutcome of primary pulmonary hyperten sion. Br Heart J 1986;55:449-58.

17 Fishman AJ, Moser KM, Fedulla PF. Perfusion lung scans $v s$. pulmonary angiography in evaluation of suspected

18 Moser KM, Daily PO, Peterson K, et al. Thromboendarterectomy for chronic, major-vessel thromboembolic pulterectomy for chronic, major-vessel thromboembolic pul-

19 Chitwood WR, Lyerly HK, Sabiston DC. Surgical management of chronic pulmonary embolism. Ann Surg 1985;201:11-26.

20 Mills SR, Jackson DC, Older RA, Heaston DK, Moore AV The incidence, etiologies and avoidance of complications of pulmonary angiography in a large series. Radiology 1980;136:295-9.

21 Nicod $\mathrm{P}$, Peterson $\mathrm{K}$, Levine $\mathrm{M}$, et al. Pulmonary angiogra phy in severe chronic pulmonary hypertension. Ann Intern Med 1987;107:565-8.

22 Gray HH, Morgan JM, Paneth M, Miller GAH. Pulmonary embolectomy for acute massive pulmonary embolism: An analysis of 71 cases. Br Heart $J$ 1988;60:196-200.

23 Anderson G, Reid L, Simon G. The radiographic appearances in primary and in thromboembolic pulmonary hypertension. Clin Radiol 1973;24:113-20.

24 Rich S, Levy PS. Characteristics of surviving and non surviving patients with primary pulmonary hypertension. Am J Med 1984;76:573-8.

25 Jones DK, Higenbottam TW, Wallwork J. Treatment of primary pulmonary hypertension with intravenous epoprostenol (prostacyclin). Br Heart J 1987;57:270-8.

26 Rozkovec A, Stradling JR, Shepherd G, MacDermot J, Oakley CM, Dollery CT. Prediction of favourable responses to long term vasodilator treatment of pulmonary hypertension by short term administration of epoproshypertension by short term administration of epoprostenol (prostacyclin

27 Rich S, Brundage BH, Levy PS. The effect of vasodilator therapy on the clinical outcome of patients with primar pulmonary hypertension. Circulation 1985;71:1191-6.

28 Greenfield LJ. Current indications for the results of Greenfield filter placement. J V asc Surg 1984;1:502-4.

29 Haissaguerre M, Bonnet J, Douvier JJ, Bricaud H. Comparison of fibrinolytic treatment with interruption of the inferior caval vein in the prevention of pulmonary embolism. Int $J$ Cardiol 1986;12:213-21.

30 Bomalski JS, Martin GJ, Hughes RL, Yao JST. Inferio vena cava interruption in the management of pulmonary embolism. Chest 1982;82:767-74.

31 Mansour M, Chang AE, Sindelar WF. Interruption of the inferior vena cava for the prevention of recurrent pulmonary embolism. Am Surg 1985;51:375-80. 